\title{
ACTIVIDAD REPELENTE DE ESPECIES VEGETALES QUE CRECEN EN LA REGIÓN DEL CHOCÓ
}

Fecha de recepción: 5 de septiembre de 2014 • Fecha de aceptación: 13 de octubre de 2014

\section{REPELLENT ACTIVITY OF VEGETAL SPECIES GROWN IN CHOCÓ REGION}

\author{
Nayive Pino Benitez ${ }^{1,3,4}$, Karina Caballero Gallardo ${ }^{2,3}$, Carlos Mario Valencia 1,3
}

\section{RESUMEN}

El objetivo de este trabajo fue el de evaluar la actividad repelente de varios extractos totales obtenidos de la fracción soluble en etanol al 96\%, de hojas de plantas provenientes del departamento del Chocó, contra adultos de Tribolium castaneum, plaga en granos almacenados. Los ensayos in vivo de la actividad repelente fueron medidos utilizando la técnica de área de preferencia, cuantificando el porcentaje de repelencia (PR) obtenido a diferentes concentraciones de exposición. Los resultados fueron analizados mediante la prueba T- pareada para determinar diferencias significativas $(\mathrm{P}<0.05)$; la comparación entre el $\mathrm{PR}$ de los diferentes extractos fue realizado usando ANOVA, empleando el post test de Dunn's para realizar comparaciones con el control positivo. Los resultados de todos los extractos muestran un aumento en su actividad repelente proporcional al incremento de la concentración con un efecto independiente reportando como extractos más efectivos los obtenidos de Ocimun campechianum (var morada), Urera laciniata y Piper tuberculatum con PR= $93 \%, 86 \%, 85 \%$ respectivamente. Con lo anterior se muestran nuevos reportes de actividad repelente para las tres especies evaluadas, y debido a lo poco explorado de la biodiversidad chocoana, este estudio se constituye en un gran aporte a la bioprospección de la flora chocoana y aporte al desarrollo sustentable de la región.

Palabras clave: Extactos etanolicos, Tribolium castaneum, bioactividad

1 Universidad Tecnológica del Chocó, Facultad de Ciencias. Laboratorio de Productos Naturales, Bloque 6, \# 316, QuibdóChocó, Colombia.

2 Grupo de investigación en Química Ambiental y Computacional, Campus de Zaragocilla, Facultad de Ciencias Farmaceuticas,

3 Bio-Red-CO-Cenivam, UIS Bucaramanga.

4 Autor corresponsal, e-mail:nayivepino@gmail.com 


\begin{abstract}
The objective this study was to evaluate the repellent activity of some total extracts derived from plants of the department of Chocó. The repellent activity of the total extracts was tested against adult insects of Tribolium castaneum, pests of stored grains. During in vivo tests, the repellent activity was measured using the area preference technique by quantifying the obtained percentage of repellency at different exposure concentrations. The results were analyzed by paired t-test to determine significant differences $(P<0.05)$. Moreover, the comparison between the repellency percentages (RP) the different extracts was performed using analysis of variance (ANOVA) by means of Dunn's post test to assess comparisons with the positive control. The results of All the evaluated extracts showed a proportional increase of its repellent activity to the concentration increase showing an independent effect. As a result, the more effective extracts were the derived from Ocimun campechianum (var morada), Urera laciniata, and Piper tuberculatum with repellency percentages of 93\%, 86\%; and 85\% respectively. Since, Chocó biodiversity has been unexplored; this paper presents new and valuable data of repellent activity for the three evaluated species. Additionally, this article constitutes a big contribution to bio-prospecting of Chocó Flora and to the sustainable development of the region.
\end{abstract}

Key words: Etanolic extracts, Tribolium castaneum, in vivo bioactivity

\title{
INTRODUCCIÓN
}

El aumento de la producción agrícola ha generado el estudio de nuevas técnicas que permitan desarrollar un buen manejo de los granos almacenados, y así mantener el recurso para las generaciones futuras, sin afectar la salud de los consumidores, según Olivero-Verbel et al. (2009). De los factores que deterioran los granos almacenados, los insectos ocupan un lugar muy importante, ya que se estima que entre un $5 \%$ y un $35 \%$ de los granos almacenados se ven afectados por estos de acuerdo con Boxall (1991). Tribolium castaneum, es una de las principales plagas de la harina de trigo en grano (Howe, 1965); para su control han sido empleados varios productos sintéticos, para los cuales se han reportado efectos adversos en animales expuestos y humanos (Nerio et al. 2010). Algunos autores (Saini y Rodríguez, 2004; Stefanazzi et al. 2006; Dal Bello y Padín 2006), han mostrado la importancia del control de estos insectos en el almacenamiento de cereales como el maíz, el trigo, sorgo, cebada y arroz, debido a la reducción en el peso, la calidad al valor comercial, poder germinativo de la semilla, finalmente disminuyendo los volúmenes de exportación agricola.

Estos problemas han llevado a la búsqueda de alternativas de control incluidas en el desarrollo de agro- sistemas sostenibles, basados en un manejo integrado de cultivo sin alterar el equilibrio del sistema tal como lo expresan (Ducrot, 2005; Céspedes y Alarcón 2011). Entre estos métodos alternativos se encuentran sustancias derivadas de plantas que actúan como bio-controladores, sustancias estas que son producidas solo por algunas plantas y reciben el nombre de metabolitos secundarios. Entre estos se encuentran terpenos, sesquiterpenos, lignanos, alcaloides, esteroides, taninos y flavonoides. No obstante, para el control de los insectos que atacan 
a los granos almacenados se ha utilizado tradicionalmente el uso de productos químicos sintéticos; y es de conocimiento que el uso indiscriminado de estos, no solo ha generado un problema de contaminación por los desechos que se producen de la actividad devastando el medio ambiente (por su baja biodegradabilidad), sino, los que causan los grandes efectos toxicológicos que afectan animales, plantas y seres humanos, tal como lo reportan varios autores (Bourguet et al. 2000; FAO 2002; Riebeiro et al. 2003; Rosegrant et al. 2007; Céspedes y Alarcón 2011).

De acuerdo a lo anterior, los insecticidas naturales a partir de extractos vegetales constituyen una interesante alternativa para el control de estos insectos, en ese sentido la flora chocoana está inexplorada, y a nivel mundial existen pocos reportes de extractos vegetales evaluados como repelentes, dado que las investigaciones han sido dirigidas más hacia los aceites esenciales, por lo que la perspectiva en esta investigación, es aún mayor. En esta investigación se evaluó la actividad repelente de los extractos vegetales obtenidos de: Urera laciniata, Urera baccifera, Rhus aurantifolia, Siparuna guianensis, Piper tuberculatum, Passiflora quadrangularis y de Ocimum campechianum (var morada).

\section{MATERIALES Y MÉTODOS}

\section{Obtención de los extractos}

Los extractos totales de las hojas de las plantas evaluadas fueron obtenidos por la técnica de maceración en frío del material vegetal seco durante tres a cinco días, usando etanol industrial al 96\%. Se toman por separado las hojas de cada una de las siete especies vegetales mencionadas, previa selección del mismo (debe estar sano y libre de enfermedades), posteriormente las hojas son secadas a $40^{\circ} \mathrm{C}$ en un horno con aire circulante. Una vez seco el material es molinado, pesado y rotulado siguiendo la metodología descrita en Pino,
2009. El macerado es filtrado al vacío y rotaevaporado a presión reducida sucesivamente hasta eliminar el solvente orgánico. El extracto etanólico asi obtenido es pesado y guardado a $4^{\circ} \mathrm{C}$ hasta el momento de su utilización.

Insectos de la especie: Tribolium castaneum, Herbst.

Los bioensayos se realizaron utilizando insectos de la especie T. castaneum, perteneciente a la familia Coleoptera: Tenebrionidae. Este insecto plaga de granos almacenados, se conoce como gorgojo de la harina, los cuales fueron recolectados en el mercado principal de la ciudad de Cartagena, obtenidos del afrecho de maíz. Los insectos fueron ubicados en recipientes de vidrio cubiertos con tela de malla, utilizando avena molida como sustrato; fueron adaptados a las condiciones del laboratorio de Química Ambiental y Computacional de la Universidad de Cartagena, a una temperatura de $26 \pm 2^{\circ} \mathrm{C}$, humedad relativa de $70-85 \%$ y fotoperíodos 10:14h, luz: oscuridad, siguiendo parámetros reportados por OliveroVerbel et al. (2009).

\section{Bioensayos de Actividad repelente}

Los ensayos de la actividad repelente fueron realizados por el método de área de preferencia siguiendo lo descrito por Tapodjou et al. (2005) y Chaubey, (2007). Para realizar el ensayo se colocaron un total de 20 insectos adultos de T. castaneum en el interior de una caja petri con papel filtro cortado a la mitad, resultando dos áreas de trabajo, una tratada con volúmenes iguales de diferentes concentraciones de extracto disuelto en acetona, y la otra área del papel filtro solo con acetona; las concentraciones resultantes fueron 100, 150, 200, 250 y $300 \mu \mathrm{g} / \mathrm{mL}$. Como control positivo fue utilizado una formulación repelente comercial de nombre Stay off, la cual contiene como ingrediente activo etil-butil-acetil-aminopropianato al 15\% (IR3535), el cual fue evaluado de igual forma que 
los extractos, generando 5 concentraciones de ingrediente activo por unidad de área, el testigo solo se trato con el disolvente. Según la EPA, El IR3535 es un repelente sintético seguro, no es peligroso cuando es inhalado o usado sobre la piel; pero si cae en los ojos podría causar algún tipo de irritación. El IR3535 es químicamente similar al aminoácido $\beta$-alanina. Por su naturaleza química y baja toxicidad, la EPA lo clasifica como un bioplaguicida (O'Farrill-Nieves, 2008).

Tanto las áreas tratadas con el extracto etanólico como el control $(0.5 \mathrm{~mL} \mathrm{c} / \mathrm{u})$ fueron secadas a temperatura ambiente, por un período de 10 minutos con el objetivo de permitir la evaporación del solvente, de la caja de Petri con ayuda de un vial. Los recuentos de gorgojos presentes en cada mitad del papel filtro fueron realizados después de 2 y 4 horas de exposición.

Para la determinación del porcentaje de repelencia (PR) fue utilizada la siguiente ecuación siguiendo los parámetros descritos por (Tapondjou et al. 2005):

$P R=\left[\left(N_{c}-N_{t}\right) /\left(N_{c}+N_{t}\right)\right] \times 100$

Donde:

$\mathrm{N}_{c}$ : equivale al número de insectos en el área control (acetona).

$N_{t}$ : equivale al número de insectos en el área tratada (E+ acetona).

De acuerdo a lo anterior, los insecticidas naturales a partir de extractos vegetales constituyen una interesante alternativa para el control de estos insectos, en ese sentido la flora chocoana está inexplorada.

para luego ser nuevamente pegadas con cinta adhesiva y colocadas en la caja de Petri. De forma inmediata fueron colocados 20 insectos adultos de T. castaneum, de diferente sexo en la mitad del papel, con ayuda de un vial. Seguidamente, la caja fue tapada y almacenada en condiciones de oscuridad continua (Chaubey, 2007). Para cada concentración de extracto vegetal fueron realizadas 3 réplicas y cada ensayo fue repetido dos veces.

Las áreas tratadas y no tratadas fueron dejadas por 10 minutos al aire libre con el objeto de que el solvente se evaporara del papel filtro. Luego fueron unidas con cinta transparente. Posteriormente fueron liberados 20 gorgojos adultos en el centro
En caso de que sea encontrado mayor número de organismos en el área tratada $\left(N_{t}>N_{c}\right)$, el porcentaje de repelencia es considerado negativo, e interpretado como actividad atrayente.

\section{Análisis de los datos}

Para cada extracto los resultados se presentan como la media del porcentaje de repelencia (PR) \pm error estándar $(\mathrm{X} \pm \mathrm{SE})$. El número promedio de insectos en cada mitad del papel filtro fue comparada mediante la prueba T-pareada para determinar diferencias significativas $(P<0.05)$. El signo obtenido en el cálculo de PR fue empleado para cualificar la acción repelente (positivo) o atrayente (negativo) de 
los extractos vegetales. En todos los casos, la distribución normal y homogeneidad de varianzas fue evaluada previamente por los test de KolmogorovSmirnov y Bartlett, respectivamente. Comparación entre el PR de los diferentes extractos fue realizado usando ANOVA, empleando el post test de Dunn's para valorar comparaciones con el control positivo. Todos estos procedimientos fueron realizados con el software Graphpad Instat.

\section{RESULTADOS Y DISCUSIÓN}

En el laboratorio de química ambiental y computacional de la universidad de Cartagena, con experiencia en la evaluación de actividad repelente de aceites esenciales contra la especie $T$. castaneum, se implementó y valido la técnica para el ensayo con extractos etanólicos, cuyos resultados para los extractos y el repelente comercial IR3535 durante de 2 y 4 horas de exposición se muestran en la Tabla 1.

En este estudio, todos los extractos evaluados fueron activos a partir de la concentración de 200 $\mu \mathrm{g} / \mathrm{mL}$, cuyo RP iba en aumento a medida que aumentaba la concentración, igual lo reporta Novo et al. (1998), en trabajos con extractos de Aloysia polistachya, donde el porcentaje de repelencia aumenta con la concentración, también muestra que la repelencia varía de acuerdo al solvente usado, siendo más efectivo el hexano que el etanol. Resultados parecidos muestran Pascual-Villalobos, (1998) y Gutiérrez et al., (2008).

Aunque diversos estudios han mostrado bioactividad (antimicrobianas y antioxidantes) en los extractos vegetales estudiados, (Pino, 2009; Pino y Stashenko, 2009; Pino et al. 2009), la actividad repelente no se había realizado. Asimismo ocurre para las Ocimun spp (Sanchez et al. 2000; Fernández et al. 2007 y Runyoro et al. 2010), y para las Siparuna spp, (Ciccio y Gómez, 2002).
Tabla 1. Porcentaje de repelencia e intervalos de confianza de los extractos vegetales y el repelente comercial (IR3535) frente a Tribolium castaneum.

\begin{tabular}{|c|c|c|c|}
\hline \multirow[t]{2}{*}{ ESPECIE } & \multirow{2}{*}{$\begin{array}{l}\text { Concen- } \\
\text { tracio- } \\
\text { nes ( } \mu \mathrm{g} / \\
\mathrm{mL})\end{array}$} & \multicolumn{2}{|c|}{$\begin{array}{c}\text { \% de Repelencia } \\
\text { según el tiempo } \\
\text { de exposición }\end{array}$} \\
\hline & & 2 horas & 4 horas \\
\hline \multirow{5}{*}{$\begin{array}{c}\text { Urera } \\
\text { baccifera }\end{array}$} & 100 & $45 \pm 4^{*}$ & $-3 \pm 24$ \\
\hline & 150 & $61 \pm 4^{\star a}$ & $50 \pm 6^{* a}$ \\
\hline & 200 & $66 \pm 2^{\star}$ & $55 \pm 6^{\star}$ \\
\hline & 250 & $70 \pm 2^{*}$ & $70 \pm 3^{*}$ \\
\hline & 300 & $76 \pm 2^{*}$ & $73 \pm 3^{\star}$ \\
\hline \multirow{5}{*}{$\begin{array}{l}\text { Urera } \\
\text { laciniata }\end{array}$} & 100 & $6 \pm 21$ & $25 \pm 10$ \\
\hline & 150 & $51 \pm 8 *$ & $48 \pm 6^{*}$ \\
\hline & 200 & $66 \pm 6^{*}$ & $63 \pm 5^{\star}$ \\
\hline & 250 & $75 \pm 2^{*}$ & $70 \pm 2^{\star}$ \\
\hline & 300 & $86 \pm 2^{*}$ & $80 \pm 4^{\star}$ \\
\hline \multirow{5}{*}{$\begin{array}{c}\text { Rhus } \\
\text { aurantifolia }\end{array}$} & 100 & $-10 \pm 12$ & $10 \pm 20$ \\
\hline & 150 & $1 \pm 23$ & $40 \pm 8 *$ \\
\hline & 200 & $60 \pm 2^{\star}$ & $56 \pm 2^{\star}$ \\
\hline & 250 & $66 \pm 11 *$ & $67 \pm 6^{*}$ \\
\hline & 300 & $75 \pm 3^{\star}$ & $71 \pm 7^{*}$ \\
\hline \multirow{5}{*}{$\begin{array}{l}\text { Siparuna } \\
\text { guianensis }\end{array}$} & 100 & $-13 \pm 11$ & $-28 \pm 9$ \\
\hline & 150 & $8 \pm 8$ & $-4 \pm 5$ \\
\hline & 200 & $21 \pm 8$ & $23 \pm 5$ \\
\hline & 250 & $41 \pm 4^{*}$ & $43 \pm 3^{\star}$ \\
\hline & 300 & $74 \pm 3^{*}$ & $73 \pm 5^{\star}$ \\
\hline \multirow{5}{*}{$\begin{array}{c}\text { Ocimum } \\
\text { campechianum } \\
\text { variedad } \\
\text { morada }\end{array}$} & 100 & $45 \pm 8^{*}$ & $51 \pm 9 *$ \\
\hline & 150 & $65 \pm 9 * a$ & $63 \pm 7 * a$ \\
\hline & 200 & $75 \pm 3^{\star a}$ & $70 \pm 0$ *a \\
\hline & 250 & $80 \pm 3^{*}$ & $76 \pm 4^{\star}$ \\
\hline & 300 & $93 \pm 2^{*}$ & $83 \pm 5^{\star}$ \\
\hline
\end{tabular}




\begin{tabular}{|c|c|c|c|}
\hline \multirow{5}{*}{$\begin{array}{c}\text { Piper } \\
\text { tuberculatum }\end{array}$} & 100 & $13 \pm 16$ & $-5 \pm 4$ \\
\hline & 150 & $65 \pm 10^{*}$ & $44 \pm 18 *$ \\
\hline & 200 & $76 \pm 4^{\star}$ & $50 \pm 8^{*}$ \\
\hline & 250 & $80 \pm 5^{\star}$ & $60 \pm 10^{*}$ \\
\hline & 300 & $85 \pm 6^{*}$ & $70 \pm 6^{*}$ \\
\hline \multirow{5}{*}{$\begin{array}{c}\text { Passiflora } \\
\text { quadrangu- } \\
\text { laris }\end{array}$} & 100 & $8 \pm 16$ & $5 \pm 10$ \\
\hline & 150 & $21 \pm 19$ & $23 \pm 15$ \\
\hline & 200 & $33 \pm 16^{*}$ & $28 \pm 29$ \\
\hline & 250 & $51 \pm 18^{*}$ & $46 \pm 6^{*}$ \\
\hline & 300 & $56 \pm 14^{\star}$ & $56 \pm 11$ * \\
\hline \multirow{5}{*}{ IR 3535} & 100 & $48 \pm 7^{\star}$ & $48 \pm 6^{\star}$ \\
\hline & 150 & $53 \pm 11$ * & $13 \pm 6$ \\
\hline & 200 & $65 \pm 10^{*}$ & $61 \pm 7^{*}$ \\
\hline & 250 & $73 \pm 8^{\star}$ & $75 \pm 4^{\star}$ \\
\hline & 300 & $83 \pm 2^{\star}$ & $80 \pm 3^{\star}$ \\
\hline
\end{tabular}

1. Promedio de los porcentajes de repelencia a diferentes tiempos durante 2 y 4 horas de exposición, los valores son \pm SEM de 6 réplicas. *. Diferencia significativa entre el número de organismos en ambas áreas tratadas y no tratadas, usando una Prueba $T$ pareada $(P<0.05)$.

a. Diferencia significativa entre los extractos y el control (IR3535), usando ANOVA, con post-test Dunn's.

Son escasos los estudios de repelencia en extractos vegetales frente a T. castaneum, más frecuentes son los estudios con aceites esenciales como lo muestra en su revisión Nerio et al. 2010, no obstante, sus comportamientos frente a los PR son parecidos con la concentración que muestran los aceites esenciales (Caballero-Gallardo et al. 2011; Espitia, 2011).

Dado que los resultados del estudio muestran que los extractos obtenidos de las especies Ocimun campechianum (var morada), Urera laciniata y Piper tuberculatum a las dos horas de exposición presentaron para la concentración de $300 \mu \mathrm{g} / \mathrm{mL}$, un porcentaje de repelencia (PR) de 93\%, 86\% y $85 \%$ respectivamente, mayor al del control positivo (83\%),
Aunque diversos estudios han mostrado bioactividad (antimicrobianas y antioxidantes) en los extractos vegetales estudiados, (Pino, 2009; Pino y Stashenko, 2009; Pino et al. 2009), la actividad repelente no se había realizado.

fueron, los más promisorios, ya que producen un gran efecto repelente sobre la especie T. castaneum y este se mantiene con relación al tiempo que fue evaluado, lo cual indica que podrían ser utilizados en el control de estos organismos tan perjudiciales para la agroindustria.

\section{AGRADECIMIENTOS}

El presente trabajo fue realizado gracias al apoyo brindado por la Universidad de Cartagena, especialmente el Dr. Jésus Olivero-Vervel, Vicerrector de Investigaciones y al Patrimonio autónomo para la Ciencia la Tecnología y la Innovación, Francisco José de Caldas, contrato RC- 0572-2012. 


\section{REFERENCIAS}

1. Bourguet D, Genissel A, Raymond M. 2000. Insecticide resistance and dominance levels. Journal of Economic Entomology, 93:1588-1595.

2. Boxall RA. 1991. Post-harvest losses to insect: A world overview. In Biodeterioration and Biodegradation 8. H.W. Rossmooore, ed. Elsevier, London, UK. 95p

3. Caballero-Gallardo K, Olivero-Verbel J, Stashenko E. 2011. Repellent activity of essential oils and some of their individual constituents against Tribolium castaneum Herbst. Journal of Agricultural and Food Chemistry. 59:1690-1696.

4. Céspedes C, Alarcón J. 2011. Biopesticidas de origen botánico, fitoquímicos y extractos de Celastraceae, Rhamnaceae y Scrophulariaceae. Boletín Latinoamericano y del Caribe de Plantas Medicinales y Aromáticas. 10:75-181

5. Chaubey MK, 2007. Insecticidal activity of Trachyspermum ammi (Umbelliferae), Anethum graveolens (Umbelliferae) and Nigella sativa (Ranunculaceae) essential oils against storedproduct beetle Tribolium castaneum Herbst (Coleoptera: Tenebrionidae). African Journal of Agricultural Ressearch, 2:596-600

6. Ciccio J, Gómez L. 2002. Volatils constituents of hte leaves of Siparuna thecaphora (Siparunaceae) from Turrialba, Costa Rica. Revista de Biología tropical, 50: 963-967

7. Dal Bello G, Padín S. 2006. Olfatometro simple para evaluar la actividad biológica de aleloquímicos vegetales en Tribolium castaneum, Herbst. Agrociencia, 2:23-26

8. Ducrot PH. 2005. Organic chemistry's contribution to the understanding of biopesticida activity of natural products from higher plants. pp. 47-58. In: Regnault, R.C., B.J.J. Philogene y C. Vincent (eds.). Biopesticides of plant origin. Lavoiser and Intercept, Ltd., Paris and Andover. $313 \mathrm{p}$.

9. Espitia C R. 2011, Evaluación de la actividad repelente e insecticida de aceites esenciales extraídos de plantas aromáticas utilizados contra Tribolium castaneum Herbst (Coleoptera: Tenebrionidae). Tesis para optar el titulo de MSc. Universidad Nacional de Colombia Facultad de Medicina, Departamento de Toxicología. Grupo de Química Ambiental y Computacional, Universidad de Cartagena, Colombia, 61p

10. FAO/ 2002. Países Bajos sobre el Carácter Multifuncional de la Agricultura y la Tierra. En: http://www.fao.org/docrep/X2777S/X2777S00. htm. Consulta: Abril de 2012

11. Fernández K, Patiño A., Murillo E \& Méndez J. 2007. Actividad antioxidante y antimicrobial de los volátiles de cuatro variedades de albahacas cultivadas en el departamento del Tolima. Scientia et Technica, XIII (33): 401-403

12. Gutiérrez FS, Stefanazzi N, Purray AP, Ferrero AA. 2008. Bioactividad de extractos de hojas de aloysia polystachya (verbenaceae), en larvas y adultos de Tribolium castaneum, Herbst. Boletín de Sanidad Vegetal. Plagas, 34:501-508

13. Howe RW. 1965. Losses caused by insects and mites in stored foods and foodstuffs. Nutrition Abstracts and Reviews, 35:285-302.

14. Nerio L, Olivero J, Stashenko E. 2010. Repellent activity of essential oils: A review. Bioresource Technology, 101:372-378

15. Novo RJ, Viglianco A, Nassetta M.1998. Actividad repelente de diferentes extractos 
vegetales sobre Tribolium castaneum (Herbst), Agriscientia, 14:31-36.

16. O'Farrill-Nieves H. 2008. Las Plagas del Hogar y el Jardín. Departamento de Protección de Cultivos. Ediciones: Universidad de Puerto Rico. Número 8, páginas 1-2

17. Olivero-Verbel J, Caballero K, Jaramillo B, Stashenko E. 2009. Actividad repelente de los aceites esenciales de Lippia origanoides, Citrus sinensis y Cymbopogon nardus cultivadas en Colombia frente a Tribolium castaneum, Herbst. Salud UIS, 41:244-250

18. Olivero-Verbel J, Nerio LS, Stashenko EE. 2010. Bioactivity against Tribolium castaneum Herbst (Coleoptera: Tenebrionidae) of Cymbopogon citratus and Eucalyptus citriodora essential oils grown in Colombia. Pest Management Science, 66:664-668

19. Pascual-Villalobos, M.J. 1998. Repelencia, inhibición del crecimiento y toxicidad de extractos vegetales en larvas de Tribolium castaneum, (Herbst). (Coleptera:Tenebrionidae). Boletín de Sanidad Vegetal. Plagas, 24:143-154

20. Pino N, Estashenko E. 2009. Antibiotic validation of medicinal plants of the Northwest of Colombia against Staphylococcus aureus. Boletín Latinoamericano y del Caribe de Plantas Medicinales y Aromáticas. 8:145-150

21. Pino N, Meléndez EM, Stashenko EE. 2009. Eugenol and methyl eugenol chemotype of essential oil of species Ocimum gratissimum L. and Ocimum campechianum Mill., from the northwest region of Colombia. Journal of Chromatographic Science, 47:800-803
22. Pino N. 2009. Plantas útiles del departamento del Chocó, Parte I Extractos. Universidad Tecnológica del Chocó. Editorial Uryco MedellínColombia, 312p.

23. Riebeiro BM, Guedes RNC, Oliveira EE, Santos JP. 2003. Insecticide resistance and synergism in Brasilian populations of Sitophiluszeamais (Coleoptera: Curculionidae). Journal of Stored Products Research, 39:21-31.

24. Runyoro D, Ngasssapa $O$, Vagionas K, Aligiannis N, Graikou K, Chinou I. 2010. Chemical composition and antimicrobial activity of the essential oils of four Ocimun species growing in Tanzania. Food chemistry. 199:311-316.

25. Saini E, Rodríguez SM. 2004. Insectos perjudiciales a los productos almacenados. Universidad de Chile. Publicación del Instituto de Microbiología y Zoología, 56p.

26. Sánchez E, Leal I, Fuentes L. Rodríguez C. 2000. Estudio farmacognóstico de Ocimum basilicum L. (albahaca Blanca). Revista Cubana de Farmacia, 4:187-195.

27. Stefanazzi MM, Gutiérrez T, Standler NA, Bonni A, Ferrero A. 2006. Actividad biología del aceite esencial de Tagetes termiflora kunth (Asteraceae) en Tribolium castaneum, Herbst (Insecta, coleoptera, tenebrionidae). Boletín de Sanidad Vegetal. Plagas, 32:439-447

28. Tapondjou AL, Ader C, Fontem DA, Bouda H, \& Reichmut C. 2005. Bioactivities of cimol and essential oils of Cupressus sempervivens and Eucaliptus saligna against Sitophilus Zea mays Motschul Sky and Tribolium confusum duVal. Journal of Stored Products Research, 41:91-102. 